\title{
Multichannel frequency and time-frequency analysis
}

\author{
Koenig, T ; Pascual-Marqui, R D
}

DOI: https://doi.org/10.1017/CBO9780511596889.008

Posted at the Zurich Open Repository and Archive, University of Zurich ZORA URL: https://doi.org/10.5167/uzh-27915

Book Section

Originally published at:

Koenig, T; Pascual-Marqui, R D (2009). Multichannel frequency and time-frequency analysis. In: Michel, C M; Koenig, T; Brandeis, D; Gianotti, L R R; Wackermann, J. Electrical Neuroimaging. Cambridge: Cambridge University Press, 145-168.

DOI: https://doi.org/10.1017/CBO9780511596889.008 
Cambridge University Press

978-0-521-87979-8 - Electrical Neuroimaging

Edited by Christoph M. Michel, Thomas Koenig, Daniel Brandeis, Lorena R. R. Gianotti and Jiri Wackermann

Frontmatter

More information

\section{Electrical Neuroimaging}


Cambridge University Press

978-0-521-87979-8 - Electrical Neuroimaging

Edited by Christoph M. Michel, Thomas Koenig, Daniel Brandeis, Lorena R. R. Gianotti and Jiri

Wackermann

Frontmatter

More information

\section{Electrical Neuroimaging}

Edited by

Christoph M. Michel

Thomas Koenig

Daniel Brandeis

Lorena R.R. Gianotti

Jiří Wackermann 
Cambridge University Press

978-0-521-87979-8 - Electrical Neuroimaging

Edited by Christoph M. Michel, Thomas Koenig, Daniel Brandeis, Lorena R. R. Gianotti and Jiri

Wackermann

Frontmatter

More information

CAMBRIDGE UNIVERSITY PRESS

Cambridge, New York, Melbourne, Madrid, Cape Town,

Singapore, São Paulo, Delhi

Cambridge University Press

The Edinburgh Building, Cambridge CB2 8RU, UK

Published in the United States of America

by Cambridge University Press, New York

www.cambridge.org

Information on this title: www.cambridge.org/9780521879798

C) Cambridge University Press 2009

This publication is in copyright. Subject to statutory exception and to the provisions of relevant collective licensing agreements, no reproduction of any part may take place without

the written permission of Cambridge University Press.

First published 2009

Printed in the United Kingdom

at the University Press, Cambridge

A catalogue record for this publication is available from the British Library

Library of Congress Cataloguing in Publication data

Electrical neuroimaging / edited by Christoph M. Michel... [et al.].

p. $; \mathrm{cm}$.

Includes bibliographical references and index.

ISBN 978-0-521-87979-8 (hardback)

1. Brain - Imaging. 2. Electroencephalography.

3. Evoked potentials (Electrophysiology) I. Michel, Christoph M., 1959- II. Title.

[DNLM: 1. Brain - physiology. 2. Brain Mapping - methods.

3. Electroencephalography - methods.

4. Evoked Potentials. WL 335 E38 2009]

QP376.6.E44 2009

$616.8^{\prime} 047547-\mathrm{dc} 22 \quad 2009009343$

ISBN 978-0-521-87979-8 hardback

Cambridge University Press has no responsibility for the persistence or accuracy of URLs for external or third-party Internet websites referred to in this publication, and does not guarantee that any content on such websites is, or will remain, accurate or appropriate.

Every effort has been made in preparing this publication to provide accurate and up-to-date information which is in accord with accepted standards and practice at the time of publication. Although case histories are drawn from actual cases, every effort has been made to disguise the identities of the individuals involved. Nevertheless, the authors, editors and publishers can make no warranties that the information contained herein is totally free from error, not least because clinical standards are constantly changing through research and regulation. The authors, editors and publishers therefore disclaim all liability for direct or consequential damages resulting from the use of material contained in this publication. Readers are strongly advised to pay careful attention to information provided by the manufacturer of any drugs or equipment that they plan to use. 
Cambridge University Press

978-0-521-87979-8 - Electrical Neuroimaging

Edited by Christoph M. Michel, Thomas Koenig, Daniel Brandeis, Lorena R. R. Gianotti and Jiri Wackermann

Frontmatter

More information

\section{Dedicated to}

Dietrich Lehmann, our teacher, mentor and friend 


\section{Contents}

List of contributors viii

Preface ix

1 From neuronal activity to scalp potential fields 1 Daniel Brandeis, Christoph M. Michel and Florin Amzica

\section{Scalp field maps and their} characterization 25

Thomas Koenig and Lorena R.R. Gianotti

3 Imaging the electric neuronal generators of EEG/MEG 49 Roberto D. Pascual-Marqui, Kensuke Sekihara, Daniel Brandeis and Christoph M. Michel

4 Data acquisition and pre-processing standards for electrical neuroimaging 79 Christoph M. Michel and Daniel Brandeis

\section{Overview of analytical} approaches 93

Thomas Koenig and Jiří Wackermann

6 Electrical neuroimaging in the time domain 111 Christoph M. Michel, Thomas Koenig and Daniel Brandeis
7 Multichannel frequency and time-frequency analysis 145 Thomas Koenig and Roberto D. Pascual-Marqui

8 Statistical analysis of multichannel scalp field data

Thomas Koenig and Lester Melie-García

9 State space representation and global descriptors of brain electrical activity 191 Jiří Wackermann and Carsten Allefeld

10 Integration of electrical neuroimaging with other functional imaging methods 215 Daniel Brandeis, Christoph M. Michel, Thomas Koenig and Lorena R.R. Gianotti

Index 


\section{Contributors}

\section{Carsten Allefeld}

Institute for Frontier Areas of Psychology and Mental Health

Freiburg im Breisgau, Germany

Florin Amzica

Department of Stomatology

Université de Montreal

Montreal, Canada

\section{Daniel Brandeis}

Department of Child and Adolescent

Psychiatry

University of Zürich

Switzerland

and Central Institute of Mental Health

Mannheim, Germany

\section{Lorena R.R. Gianotti}

The KEY Institute for Brain-Mind Research

University Hospital of Psychiatry

University of Zürich

Zürich, Switzerland

\section{Thomas Koenig}

Department of Psychiatric

Neurophysiology

University Hospital of Psychiatry

University of Bern

Bern, Switzerland

\section{Lester Melie-García}

Neuroinformatics Department

Cuban Neuroscience Center

Havana, Cuba

\section{Christoph M. Michel}

Functional Brain Mapping Laboratory

Neurology Clinic, University Hospital

and Fundamental Neuroscience

Department

University Medical School

University of Geneva

Geneva, Switzerland

\section{Roberto D. Pascual-Marqui}

The KEY Institute for Brain-Mind Research University Hospital of Psychiatry

University of Zürich

Zürich, Switzerland

\section{Kensuke Sekihara}

Department of Systems Design and

Engineering

Tokyo Metropolitan University

Tokyo, Japan

\section{Jiří Wackermann}

Institute for Frontier Areas of Psychology

and Mental Health

Freiburg im Breisgau, Germany 
Cambridge University Press

978-0-521-87979-8 - Electrical Neuroimaging

Edited by Christoph M. Michel, Thomas Koenig, Daniel Brandeis, Lorena R. R. Gianotti and Jiri

Wackermann

Frontmatter

More information

\section{Preface}

In 1929, Hans Berger, the founding father of electroencephalography (EEG), described EEG as a "window into the brain," because EEG appeared to be a sensitive indicator of mental states. Eighty years later, recording and analysis methods exist that have made EEG a widespread and validated tool to observe the spatial and temporal dynamics of brain network activity during a large variety of mental states and processes in a completely noninvasive fashion. This has been made possible by significant technological advances that now allow the simultaneous recording of an EEG from a large number of electrodes at a high sampling rate, and the application of space-domain oriented approaches to the analysis of these recordings. This book gives an overview of these methods. Illustrated by various examples from experimental and clinical studies, the book is a tutorial on how to use EEG as a modern functional imaging method with the advantage of directly recording neuronal activity with millisecond temporal resolution, an approach called electrical neuroimaging.

Electrical neuroimaging has enormous potential if properly applied, but it can also easily lead to erroneous conclusions if its basic principles are not properly understood. This book intends to give a comprehensive introduction to the basics of multichannel recording of EEG and event-related potential (ERP) data, and to spatio-temporal analysis of the potential fields. All chapters include practical examples from clinical and experimental research. The book enables a researcher to measure valid data, select and apply appropriate analysis strategies and draw the correct conclusions when analyzing and interpreting multichannel EEG/ERP data. It informs the research community about the possibilities opened by these space-domain oriented strategies to the analysis of brain electrical activity, and of their potential for multimodal integration with other (structural, metabolic, etc.) data.

Electrical neuroimaging is decisively different from the traditional analysis of EEG and ERP data, which is based upon waveform morphology and/or frequency characteristics of recordings at certain electrode positions. Electrical neuroimaging exclusively considers the spatial properties of electric fields and the temporal dynamics of these fields. It uses these to conclude on putative generators in the brain that gave rise to these recorded fields on the scalp. The approach therefore fills an important gap that has not been covered by other comprehensive textbooks on the analysis of EEG and ERP.

The book begins with an introduction of what the EEG on the scalp surface actually records. It comprises the basics of what we know about the generation of electric fields in the brain, which can be measured noninvasively at the scalp, and about the generation of oscillatory activity at different frequency ranges. This basic knowledge is needed for understanding the following chapter on how electric potential fields on the scalp are generated and how they are characterized. Knowing the generators in the brain and the expression of these activities on the scalp surface allows the key issue of estimating putative generators in the brain to be addressed. The book therefore contains a thorough discussion of the methods available to solve the electromagnetic inverse problem, focusing mainly on distributed inverse solutions that impose minimal a priori constraints. It is followed by a rather practical chapter on the basic requirements for recording high-density EEG and preparing these data for electrical neuroimaging. The subsequent chapters show with many practical examples how to assess the many different facets of time- and frequency-domain EEG. The knowledge and methods 
Cambridge University Press

978-0-521-87979-8 - Electrical Neuroimaging

Edited by Christoph M. Michel, Thomas Koenig, Daniel Brandeis, Lorena R. R. Gianotti and Jiri

Wackermann

Frontmatter

More information

Preface

presented here are strictly based on well-established facts about the relation between generators in the brain and scalp-surface recordings. The results obtained by these methods thus have simple and unique interpretations in terms of intracerebral brain electric activity. Finally, the emerging field of multimodal integration is discussed, with special emphasis on the combination of EEG-based neuroimaging and functional MRI.

The book will be of great utility to cognitive neuroscientists, but also clinical neurophysiologists, neurologists and psychiatrists, as we emphasize with many practical examples the usefulness of these methods for developmental and clinical applications. The first attempts in the early 1990s to introduce quantitative EEG mapping into clinical applications were hampered by severe technical difficulties. This is not the case any more. High resolution EEG with up to 256 channels is very easy and fast to apply in clinical routine in adults as well as in children, allowing the application of the analysis methods described in this book to pathological alterations of brain function that continue to intrigue clinical neurophysiologists. It should provide them with a new tool to critically increase the sensitivity, specificity and interpretability of the measure they have used for over half of a century, which is the electroencephalogram. 